\title{
Relating CYP2B6 Genotype and EFV Resistance Among Women Living With HIV With High Viremia in Uganda: A Nested Cross-Sectional Study.
}

\author{
ALLAN BUZIBYE ( $\sim$ abuzibye@idi.co.ug ) \\ Infectious Diseases Institute https://orcid.org/0000-0001-5874-3219 \\ Kara Wools-Kaloustian \\ Indiana University
}

Adeniyi Olagunju

Obafemi Awolowo University

Ellon Twinomuhwezi

Infectious Diseases Institute

Constantin Yiannoutsos

Indiana University Fairbanks School of Public Health

Andrew Owen

University of Liverpool

Megan Neary

University of Liverpool

Joshua Matovu

Infectious Diseases Institute

Grace Banturaki

Infectious Diseases Institute

Barbara Castelnuovo

Infectious Diseases Institute

Mohammed Lamorde

Infectious Diseases Institute

Saye Khoo

University of Liverpool

Catriona Waitt

Infectious Diseases Institute

Agnes Kiragga

Infectious Diseases Institute

Research 
Keywords: HIV, pregnancy, post-partum, CYP2B6, HIV drug resistance, single nucleotide polymorphisms Posted Date: April 13th, 2021

DOI: https://doi.org/10.21203/rs.3.rs-401314/v1

License: (c) (1) This work is licensed under a Creative Commons Attribution 4.0 International License. Read Full License 


\section{Abstract}

\section{Background}

We investigated the association between CYP2B6 polymorphisms and efavirenz drug resistance among women living with HIV started on anti-retroviral therapy during pregnancy and with high viremia during post-partum.

\section{Methods}

This was a cross sectional study. Women between 6-12 weeks post-partum with viral load $>1000$ copies/ml were eligible. Sanger sequencing to detect resistant mutations and host genotyping were performed. We categorized efavirenz metabolizer genotype according to the AIDS clinical trials group algorithm as slow, intermediate and extensive; and compared efavirenz resistance among the metabolizer genotypes.

\section{Results}

Over a one-year period (July 2017-July 2018), three hundred and thirty two women were screened of whom 112 (34.8\%) had viral load $\geq 1000$ copies/ml of whom 62 had whole blood available for genotyping. Fifty-nine of these women had both viral resistance and human host genotypic results. We observed a higher frequency of efavirenz resistance among slow metabolizers ( $47 \%$ versus $34 \%$ in extensive and $28 \%$ in intermediate, metabolizers) but due to low numbers, this was not statistically significant.

\section{Conclusions}

Our findings raise the possibility that $C Y P 2 B 6$ polymorphism may contribute to efavirenz drug resistance in women started on antiretroviral therapy during pregnancy and with high viremia in the post-partum period. If confirmed in a larger study, this would have important implications for all patients in subSaharan Africa receiving efavirenz and add further support to the changes in World Health Organization policy to switch away from efavirenz as first line antiretroviral therapy in countries with a high prevalence of CYP2B6 polymorphisms.

\section{Background}

Increased access to anti-retroviral therapy (ART) has resulted in significant progress in the prevention of transmitted HIV infections in children, with a 52\% global reduction between 2010 and 2019(1). Despite the significant reduction, HIV infection among children (0-14 years) remains four times higher than the target of 40,000 set by the UNAIDS states for 2018(1). Compared to other regions of sub-Saharan Africa, East and Southern Africa have achieved greater progress, attributed to the greater widespread access to ART among pregnant and breastfeeding mothers. However, attrition from care and inconsistent treatment adherence during pregnancy and breastfeeding are still associated with HIV vertical transmission despite 
access to ART, with 10,000 of 26,000 (38.5\%) new HIV infections in children in 2018 attributed to women not retained on treatment throughout pregnancy and breast feeding(1).

Efavirenz (EFV)-based regimens were recommended for the prevention of HIV vertical transmission through the WHO Option B+ strategy, which advocates for starting ART in all pregnant women regardless of the CD4 count and continue for life. Although in 2019, the recommended first-line option in countries with high rates of pre-treatment resistance including Uganda replaced EFV with dolutegravir (DTG) (2), EFV remains an option for patients with DTG intolerance or contraindications like diabetes or concomitant treatment with anticonvulsants. A major clinical challenge with EFV is neuropsychiatric side effects that result in poor adherence in some individuals(3). A recent systematic review and metaanalysis including 39 studies reported that perceived adverse ART reactions decreased adherence in 33 (85\%) studies, with neuropsychiatric adverse reactions resulting from EFV-containing regimens contributing to poor adherence (4). In another study, $85 \%$ of women on option B+ reported central nervous system side effects and at least one missed dose was reported by $32 \%$, with a strong association between side effect burden and ART adherence being demonstrated(5).

EFV is primarily metabolized to 8-hydroxy-EFV (8-hydroxy-EFV) by the cytochrome P450 monooxygenase enzyme system. Specifically, gene-family-2, sub-Family-B and gene-6 (CYP2B6) are key to the metabolism of EFV. Studies indicate that CYP2B6 single nucleotide polymorphisms (SNPs) increase EFV plasma concentrations (6-8). Specifically, CYP2B6 $516 \mathrm{G}>\mathrm{T}$ and $983 \mathrm{C}>\mathrm{T}$ predict clinically significant increased plasma exposure in equal magnitude. Both 516 and 983 are more frequent among individuals of African ancestry as compared to Caucasians $(8,9)$. CYP2B6 15582, also common among individuals of African ancestry predicts increased EFV plasma concentration to a lesser extent.

The AIDS Clinical Trials Group (ACTG) classifies individual EFV metabolism phenotypes as slow, intermediate and extensive basing on their genotypes. Both slow and extensive metabolism may predispose an individual to drug resistance. Slow metabolizers have higher drug concentrations which may result in toxicity $(10,11)$ leading to suboptimal adherence that may results into a long 'tail' of plasma EFV in comparison to the partner nucleotide reverse transcriptase inhibitors(NRTI) which are cleared far more quickly. Specifically, drug-related side effects have been reported to contribute to poor adherence among pregnant women(3). Extensive metabolizers are at risk of sub-therapeutic concentrations (12); both states increase the risk of developing of secondary resistance.

No study has investigated the association between CYP2B6 polymorphisms and drug resistance in HIV pregnant women with high viremia, a sub-population that is likely to increase HIV prevalence in a community through vertical transmission. We hypothesized that CYP2B6 polymorphisms increase EFV drug plasma concentrations (among slow metabolizers), increasing CNS side effects resulting into poor adherence; this could lead to drug resistance attributable to long tail of functional EFV mono-therapy. As such within this study we investigated the association between CYP2B6 polymorphisms and EFV drug resistance among pregnant women with HIV RNA viral load>1000 copies/ml. 


\section{Methods}

\section{Study Design}

This was a cross-sectional sub-study of the "Tracing non-rEtained HIV PoSitivE Pregnant Women enrolled in Option B+ and ascertaining their BableS outcomes (sTEPWISe)" study. "sTEPWISe" was a prospective cohort study which took place between July 2017 and July 2018.

\section{Population}

The sTEPWISe study aimed to trace women who initiated ART under option B+ and disengaged from care to assess the actual status of the women (some may have re-engaged through a different facility, moved district or died), and the reasons for disengagement. The study took place at the HIV clinics of Kampala City Council Authority which is supported by the Infectious Diseases Institute (IDI), Uganda. Women living with HIV (WLHIV) who initiated ART during pregnancy, and were 6-12 weeks postpartum were eligible for study, whether they were engaged or disengaged from care. A woman was defined as disengaged if she had not had any ART clinic encounter within 3 months ( 90 days) from the date of data extraction (database closure). A woman was defined as retained if she was still in care and seen three months prior to data extraction.

All retained women provided a blood sample for HIV plasma viral load testing. A random sample of retained mothers with viral failure (>1000 copies/ml a cut off for WHO definition) were subjected to genotyping tests to evaluate for resistance mutations to EFV. Women disengaged from care were contacted by telephone and later visited at home in the community to obtain consent to blood draw for viral load and for HIV testing of their babies. All women with a viral load >1000 copies/ml had HIV genotyping to identify drug resistance mutations.

\section{Laboratory studies}

Drug resistance testing was performed at the Infectious Diseases Institute Core Laboratory, Kampala, Uganda, which is accredited by the College of American Pathologists (CAP). Viral RNA was isolated from plasma samples using the Invitrogen Purelink viral RNA/DNA kit (Thermo Fisher Scientific, Dreieich, Germany). HIV-1 genotyping was performed on extracted RNA using an in-house HIV-1 genotyping assay covering the protease (PR) and reverse transcriptase (RT) with subsequent sequencing using the BigDye Terminator v3.1 Cycle Sequencing Kit (Thermo Fisher Scientific, Dreieich, Germany). Sequence analysis was performed using AB3730 capillary sequencer (Thermo Fisher Scientific, Dreieich, Germany).

Genotyping of three CYP2B6 polymorphisms that predict increased EFV exposure (516G $\rightarrow$ T [rs3745274], 983T $\rightarrow C$ [rs28399499], and 15582C $\rightarrow$ T [rs4803419]) was undertaken at the University of Liverpool by real-time PCR allelic discrimination using standard TaqMan assays. Genotypes assignment were performed on a chromo4 system (Bio-Rad Laboratories, CA, USA) and Opticon Monitor version 3.1 software (Bio-Rad Laboratories). The PCR protocol involved denaturation of DNA at $95 \circ \mathrm{C}$ for $10 \mathrm{~min}, 40$ cycles of amplification at $95 \circ \mathrm{C}$ for $15 \mathrm{~s}$ and annealing at $60 \circ \mathrm{C}$ for $1 \mathrm{~min}$. 


\section{Analysis}

Drug resistance-associated mutations in protease and reverse transcriptase gene regions were interpreted using the Stanford HIV database (HIVdb) program. To predict susceptibility to NRTIs, NNRTIs and PIs a resistance score was calculated and classified as susceptible, low, intermediate, and high-level following the Stanford HIVdb scoring system (http://hivdb.stanford.edu)(13).

Metabolizer genotype was categorized according to the ACTG algorithm as slow, intermediate and extensive. We used descriptive statistics including (frequencies, means, medians to describe the study population, while a univariable logistic regression model was used to establish the relationship between EFV resistance and the metabolizer genotypes. The analysis was conducted using STATA 15.0, Texas, USA and P-value $<0.05$ were considered statistically significant.

\section{Results}

\section{Participants}

Three hundred and twenty two (322) women were enrolled between July 2017- July 2018 by the STEPWISE study of whom 110 (34.2\%) had viral load $\geq 1000 \mathrm{copies} / \mathrm{ml}$ (Figure1). Only 62 women with viral load $\geq 1000$ copies $/ \mathrm{ml}$ had whole blood available for genotyping because the study was only amended later to store whole blood. Three patient samples failed to amplify thus generated no viral resistance results, remaining with 59 of which 10 were from women retained in care and 49 from disengaged.

The baseline characteristics of the 59 mothers studied (included in the nested study) are summarized in Table 1. The median age was 25 years (interquartile range [IQR]: 22-28), median weight was 60 kilograms (IQR: 52,-66), median CD4 T-cell count was 520 cells/mm3 (IQR: 336-625) and the median viral load was 25,562 copies/ml (IQR: 6,117-59,480). All participants were on EFV, TDF and 3TC except one participant who was on emtricitabine instead of 3TC. The genotypic frequencies population were; $29 \%$, $50 \%$ and $20 \%$ for slow, intermediate and extensive phenotypes, respectively.

Table 1: Demographic, clinical and laboratory characteristics

\begin{tabular}{|c|c|}
\hline Characteristic & Median (IQR) \\
\hline Age in years & $25(22-28)$ \\
\hline Weight, $\mathrm{Kg}$ & $60(52-66)$ \\
\hline CD4 count, cells/ $\mu \mathrm{L}$ & $520(336-645)$ \\
\hline HIV viral load, copies/ml & $25,562(6,117-59,480)$ \\
\hline
\end{tabular}


HIV drug resistance to the non-nucleoside reverse transcriptase inhibitors (NNRTI) was the most frequent, followed by the nucleoside reverse transcriptase inhibitors (NRTI). Protease inhibitors (PI) had the lowest frequency of HIV drug resistance. Figure 2 shows the resistance to NNRTIs stratified by phenotype. The prevalence of EFV drug resistance mutations was $34 \%$ (high: $29 \%$, low: $3 \%$, intermediate: $2 \%$ ). The resistance profile for nevirapine was similar to that of EFV $36 \%$ (high: $31 \%$, low: $2 \%$, intermediate: $3 \%$ ). Lower resistance profiles were observed for rilpivirine $21 \%$ (high: $3 \%$, low: 10\%, intermediate: $8 \%$ ); doravirine 20\% (high: 0\%, low: 10\%, intermediate: 10\%); and etravirine 17\% (low: 14\%, intermediate: 3\%).

Among the NRTIs, two participants had high level resistance to both emtricitabine and lamivudine. The resistance profile for the rest of the drugs was: zidovudine (low-1), stavudine (low-2), didanosine (high-1, low-2,), and tenofovir (intermediate-1).

Only one participant had low-level resistance to nelfinavir. All the participants were susceptible to the rest of the Pls.

\section{Comparison of EFV resistance and host genotype}

Table 2 shows the frequency of EFV resistance stratified by genotype. We observed a numerically higher frequency of EFV resistance among slow metabolizers (47\%) followed by extensive metabolizers (31\%) and intermediate $(28 \%)$.

\section{Table 2: Comparison of EFV resistance stratified by different genotypes}

\begin{tabular}{|llllll|}
\hline $\begin{array}{l}\text { Metabolizer } \\
\text { category }\end{array}$ & $\mathrm{N}$ & $\begin{array}{l}\text { EFV } \\
\text { resistance } \\
\mathrm{n}(\%)\end{array}$ & $\begin{array}{l}\text { Unadjusted Odds ratio } \\
(\mathrm{OR})\end{array}$ & $\begin{array}{l}95 \% \text { Confidence } \\
\text { Interval (Cl) }\end{array}$ & $\begin{array}{l}\text { P- } \\
\text { value }\end{array}$ \\
Slow & 17 & $8(47)$ & 2.33 & $0.67-8.17$ & 0.185 \\
\hline Intermediate & 29 & $8(28)$ & 1.00 & 1.00 & \\
\hline Extensive & 13 & $4(31)$ & 1.16 & $0.28-4.88$ & 0.833 \\
\hline
\end{tabular}

EFV: efavirenz. Results of three participants are missing because they failed to amplify.

\section{Discussion}

In our mixed population of Ugandan retained and disengaged women started on ART during pregnancy the prevalence of virological failure was $34.2 \%$ during postpartum. Among these, EFV resistance was found in $34 \%$ of samples available for analysis. We observed a relatively higher frequency of efavirenz resistance among slow metabolizers (47\%) compared to extensive (31\%) and intermediate (28\%). Slow metabolizers were twice as likely to experience EFV resistance compared to intermediate metabolizers though this was not statistically significant. To the best of our knowledge, we report the first study to rise the possibility of higher frequency of resistance among slow metabolizers in Africa. 
The virological failure rates observed in our study were higher than those observed in similar populations (14-16). However, unlike most published studies, the current study in addition to the retained women sought to find women who had been lost to follow up. In a South African study (17), 8.4\% of the individuals lost to follow up were not on treatment and $25.1 \%$ re-engaged after treatment interruption. The treatment interruption results in high rates of resistance, so that upon re-engagement into care (for example during a subsequent pregnancy), it is highly unlikely to attain virologic suppression with reinitiation of the first-line regimen $(18,19)$. The high virologic failure rates if not addressed are likely to increase mother to child transmission and hamper the efforts of viral suppression among individuals on ART.

High rates of drug resistance are expected in HIV patients on NNRTI with virologic failure. The observed prevalence in our population is similar to that reported from Malawi in women started on ART while pregnant, with $35 \%$ prevalence among patients with viral load $\geq 1000$ copies $/ \mathrm{ml}(14)$. However, a study undertaken in Tanzania reported higher rates of NNRTI resistance (57\%) among pregnant women started on nevirapine (20). As observed in our results, resistance to nevirapine is slightly higher than for EFV and this may explain the difference in the findings.

Numerically, slow metabolizers had the highest frequency of EFV resistance, consistent with previous studies confirming our hypothesis. We hypothesized that CYP2B6 polymorphisms increase EFV drug plasma concentrations, increasing CNS side effects resulting into poor adherence and subsequently leading to drug resistance due to long tail of EFV that mimics EFV monotherapy. The slow metabolizer genotype is known to be more common among people of African descent compared to Caucasians. Indeed, it was previously observed that Ugandans were being exposed to high EFV concentrations(21) and subsequently led to the lowering for EFV dose to $400 \mathrm{mg}$ for the African population(22). Besides the genetics, several others factors contribute to drug resistance and this might explain what is observed among the extensive and intermediate metabolizers.

In response to the alarming rates(>10\%) of pre-treatment drug resistance, WHO recently recommended the use of DTG based regimen as the preferred first line regimen in in countries with resistance rates $>10 \%$ (2). The Ugandan Ministry of Health in 2018 revised the National Health Guidelines to include the use of DTG as the preferred first line ART for adults and adolescents through informed choice(23). However, EFV remains the preferred alternative in specific circumstances $(24,25)$. Furthermore the role out of DTG in women has been slow because of the signal for neuro tube defect.

\section{Limitations and strength of the study}

Although we observed high frequency of EFV resistance among the slow metabolizers, the sample size was too small to draw a statistical conclusion.

\section{Conclusions}


Our findings have raised the possibility that through increased side effects, CYP2B6 polymorphism may contribute to reduced adherence resulting in virologic failure. Data from this study might lend further support for increased advocacy for DTG even in women of reproductive potential and in countries with a high prevalence of CYP2B6 polymorphisms.

\section{List Of Abbreviations}

EFV: Efavirenz

ART: Antiretroviral therapy

AIDS: Acquired immunodeficiency syndrome

WHO: World Health Organisation

HIV: Human immunodeficiency virus

UNAIDS: Joint United Nations Programme on HIV and AIDS

DTG: Dolutegravir

ACTG: AIDS Clinical Trials Group

NRTI: Nucleotide reverse transcriptase inhibitor

CNS: Central nervous system

RNA: Ribonucleic acid

DNA: Deoxyribonucleic acid

IDI: Infectious Diseases Institute

WLHIV: Women living with HIV

VL: Viral load

CAP: College of American Pathologists

PCR: Polymerase chain reaction

USA-United States of America

NNRTIs-Non-nucleotide reverse transcriptase inhibitors

Pls- Protease inhibitors 
TDF-Tenofovir

3TC-lamivudine

OR-odds ratio

Cl-confidence interval

IQR-interquartile range

\section{Declarations}

\section{Ethical approval and consent to participate}

Ethical approval was obtained from the Joint Clinical Research Center in Uganda, and the School of Medicine Indiana University ethics committees. All study participants of the STEPWISE study provided written informed consent.

\section{Consent for publication}

Not applicable.

\section{Availability of Data and materials}

The data that support the findings of this study are available from the STEPWISE study team but restrictions apply to availability of this data and so not publicly available. Data is however available from corresponding author upon reasonable request and with permission of the STEPWISE study team.

\section{Competing interests}

The authors declare that they have no competing interests.

\section{Funding}

Research reported in this publication was supported by the National Institute Of Allergy And Infectious Diseases (NIAID), Eunice Kennedy Shriver National Institute Of Child Health \& Human Development (NICHD), National Institute On Drug Abuse (NIDA), National Cancer Institute (NCI), and the National Institute of Mental Health (NIMH), National Institute of Diabetes and Digestive and Kidney Diseases (NIDDK) , Fogarty International Center (FIC), National Heart, Lung, and Blood Institute (NHLBI) , in accordance with the regulatory requirements of the National Institutes of Health under Award Number U01Al069911East Africa leDEA Consortium. The content is solely the responsibility of the authors and does not necessarily represent the official views of the National Institutes of Health. Allan Buzibye is currently a PhD scholar and receives research support from Fogarty International Centre, National Institute of Health (grant\# 2D43TW009771-06 "HIV and co-infections in Uganda"). 
Authors' contributions

AB-DNA extraction and genotyping, writing manuscript; GB-data management and statistical analysis; ET, $\mathrm{JM}$ and AK-collected data in the main study, proposal writing and manuscript writing; $\mathrm{ML}, \mathrm{BC}, \mathrm{SK}$ and $\mathrm{CW}$ -manuscript writing; $A K, C Y$ and $K W K$ - proposal and manuscript writing; $A O, M N$ and AdO-technical support during DNA extraction and genotyping. All authors read and approved the final manuscript.

\section{Acknowledgements}

HIV Research Trust supported Allan's training in pharmacogenetic and drug level analysis assays at the University of Liverpool. Sample processing (plasma and whole blood) and storage was done from the Infectious Diseases translational laboratory, Kampala Uganda.

\section{Authors' Information}

Infectious Diseases Institute, Makerere University, College of Health Sciences, Kampala, Uganda

Allan Buzibye, Ellon Twinomuhwezi, Joshua Matovu, Grace Banturaki, Barbara Castelnovo, Muhammed Lamorde, Catriona Waitt and Agnes Kiragga.

Indiana University, School of Medicine, Infectious Diseases, Indianapolis, Indiana, USA Kara Wools-Kaloustian.

Faculty of Pharmacy, Obafemi Awolowo University, Nigeria

Adeniyi Olagunju.

Indiana University Fairbanks School of Public Health, Indianapolis, Indiana, USA

Constantin Yiannoutsos.

Department of Pharmacology and Therapeutics, University of Liverpool, United Kingdom Andrew Owen, Megan Neary, Saye Khoo and Catriona Waitt

\section{References}

1. UNAIDS. Preventing mother to child transmission Geneva: UNAIDS Media; 2020 [Available from: https://www.unaids.org/en/keywords/preventing-mother-child-transmission.

2. WHO. Update of recommendations on first- and second-line antiretroviral regimens. Internet. Geneva: World Health Organisation; 2019 05/01/2021. Contract No.: WHO/CDS/HIV/19.15.

3. Adeniyi OV, Ajayi Al, Ter Goon D, Owolabi EO, Eboh A, Lambert JJBid. Factors affecting adherence to antiretroviral therapy among pregnant women in the Eastern Cape, South Africa. 2018;18(1):175. 
4. Li H, Marley G, Ma W, Wei C, Lackey M, Ma Q, et al. The role of ARV associated adverse drug reactions in influencing adherence among HIV-infected individuals: a systematic review and qualitative meta-synthesis. AIDS and Behavior. 2017;21(2):341-51.

5. Phillips T, Cois A, Remien RH, Mellins CA, Mclntyre JA, Petro G, et al. Self-reported side effects and adherence to antiretroviral therapy in HIV-infected pregnant women under option $\mathrm{B}+$ : a prospective study. 2016;11(10):e0163079.

6. Mukonzo JK, Röshammar D, Waako P, Andersson M, Fukasawa T, Milani L, et al. A novel polymorphism in ABCB1 gene, CYP2B6* 6 and sex predict single-dose efavirenz population pharmacokinetics in Ugandans. British journal of clinical pharmacology. 2009;68(5):690-9.

7. Kwara A, Lartey M, Sagoe KW, Rzek NL, Court MH. CYP2B6 (c. 516G $\rightarrow$ T) and CYP2A6 (* 9B and/or* 17) polymorphisms are independent predictors of efavirenz plasma concentrations in HIV-infected patients. British journal of clinical pharmacology. 2009;67(4):427-36.

8. Haas DW, Ribaudo HJ, Kim RB, Tierney C, Wilkinson GR, Gulick RM, et al. Pharmacogenetics of efavirenz and central nervous system side effects: an Adult AIDS Clinical Trials Group study. Aids. 2004;18(18):2391-400.

9. Wyen C, Hendra H, Vogel M, Hoffmann C, Knechten H, Brockmeyer NH, et al. Impact of CYP2B6 983T > C polymorphism on non-nucleoside reverse transcriptase inhibitor plasma concentrations in HIV-infected patients. Journal of antimicrobial chemotherapy. 2008;61(4):914-8.

10. Marzolini C, Telenti A, Decosterd LA, Greub G, Biollaz J, Buclin T. Efavirenz plasma levels can predict treatment failure and central nervous system side effects in HIV-1-infected patients. Aids. 2001;15(1):71-5.

11. Mukonzo JK, Okwera A, Nakasujja N, Luzze H, Sebuwufu D, Ogwal-Okeng J, et al. Influence of efavirenz pharmacokinetics and pharmacogenetics on neuropsychological disorders in Ugandan HIV-positive patients with or without tuberculosis: a prospective cohort study. BMC infectious diseases. 2013;13(1):261.

12. Sukasem C, Manosuthi W, Koomdee N, Santon S, Jantararoungtong T, Prommas S, et al. Low level of efavirenz in HIV-1-infected Thai adults is associated with the CYP2B6 polymorphism. 2014;42(3):469-74.

13. Tang MW, Liu TF, Shafer RWJI. The HIVdb system for HIV-1 genotypic resistance interpretation. 2012;55(2):98-101.

14. Hosseinipour M, Nelson JA, Trapence C, Rutstein SE, Kasende F, Kayoyo V, et al. Viral suppression and HIV drug resistance at 6 months among women in Malawi's Option B+ program: results From the PURE Malawi study. 2017;75(Suppl 2):S149.

15. Onoya D, Sineke T, Brennan AT, Long L, Fox MPJA. Timing of pregnancy, postpartum risk of virologic failure and loss to follow-up among HIV-positive women. 2017;31(11):1593.

16. Woldesenbet SA, Kufa T, Barron P, Chirombo BC, Cheyip M, Ayalew K, et al. Viral suppression and factors associated with failure to achieve viral suppression among pregnant women in South Africa. 2020;34(4):589. 
17. Etoori D, Gomez-Olive FX, Reniers G, Rice B, Renju J, Kabudula CW, et al. Outcomes After Being Lost to Follow-up Differ for Pregnant and Postpartum Women When Compared With the General HIV Treatment Population in Rural South Africa. 2020;85(2):127.

18. Meresse M, March L, Kouanfack C, Bonono RC, Boyer S, Laborde-Balen G, et al. Patterns of adherence to antiretroviral therapy and HIV drug resistance over time in the $S$ tratall ANRS 12110/ESTHER trial in C ameroon. 2014;15(8):478-87.

19. Luebbert J, Tweya H, Phiri S, Chaweza T, Mwafilaso J, Hosseinipour MC, et al. Virological failure and drug resistance in patients on antiretroviral therapy after treatment interruption in Lilongwe, Malawi. 2012;55(3):441-8.

20. Ngarina M, Kilewo C, Karlsson K, Aboud S, Karlsson A, Marrone G, et al. Virologic and immunologic failure, drug resistance and mortality during the first 24 months postpartum among HIV-infected women initiated on antiretroviral therapy for life in the Mitra plus Study, Dar es Salaam, Tanzania. BMC infectious diseases. 2015;15(1):175.

21. Mukonzo JK, Owen JS, Ogwal-Okeng J, Kuteesa RB, Nanzigu S, Sewankambo N, et al. Pharmacogenetic-based efavirenz dose modification: suggestions for an African population and the different CYP2B6 genotypes. PloS one. 2014;9(1):e86919.

22. Organization WH. WHO recommends dolutegravir as preferred HIV treatment option in all populations [news release]. Mexico City. 201922 July 2019.

23. Health UMo. Consolidated guidelines for the prevention and treatment of HIV and AIDS in Uganda. In: Health, editor. Kampala, Uganda2018. p. 207-9.

24. Kamal P, Sharma SJJotES. SUN-187 Dolutegravir Causing Diabetes. 2019;3(Supplement_1):SUN187.

25. Rwegerera GM, Rimbi M, Mudhina V, Simone MT, Sefo M, Segona B. Dolutegravir induced sub-acute hepatic failure in HIV positive treatment naïve man in Botswana.

\section{Figures}




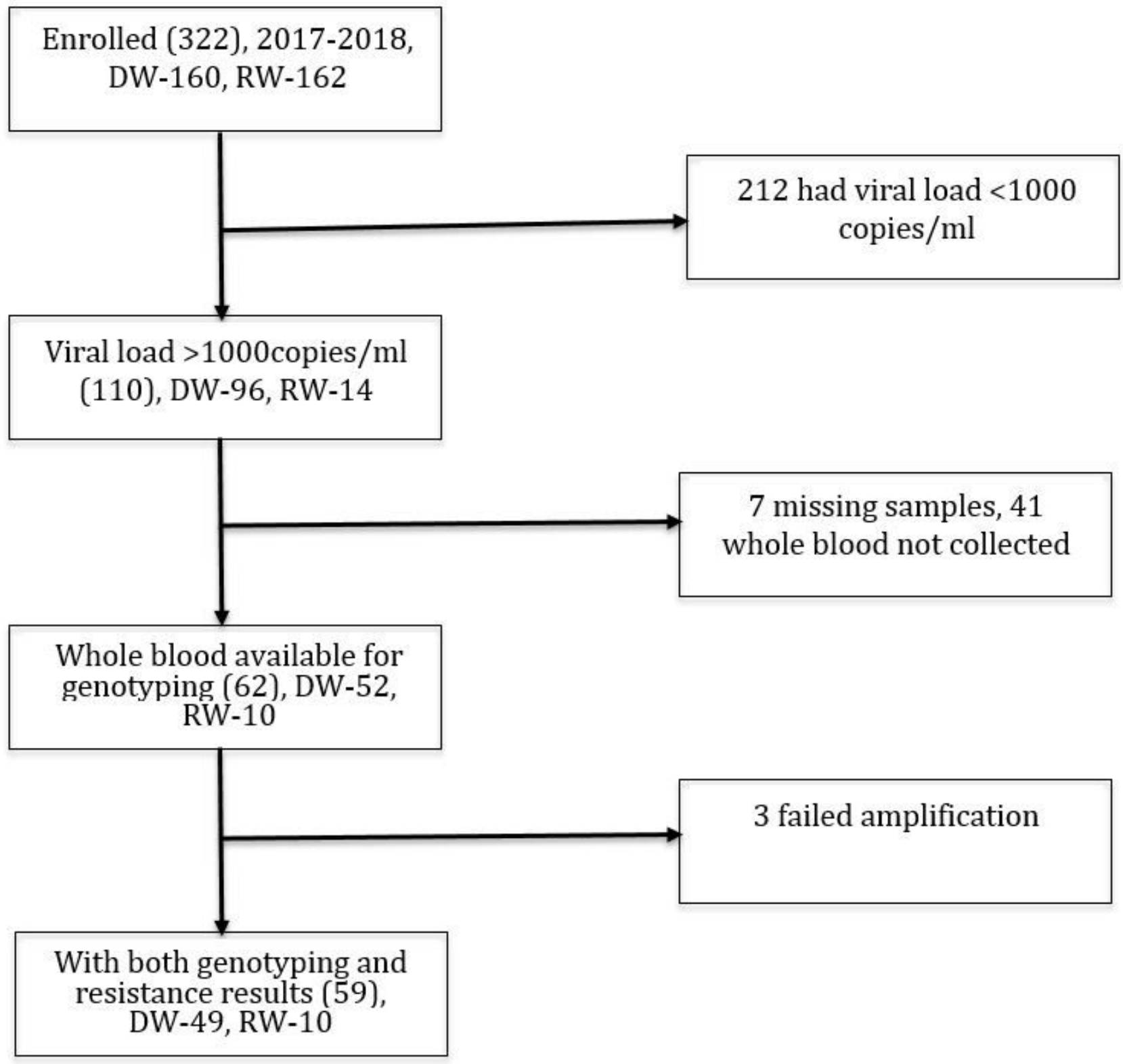

Figure 1

Study profile 


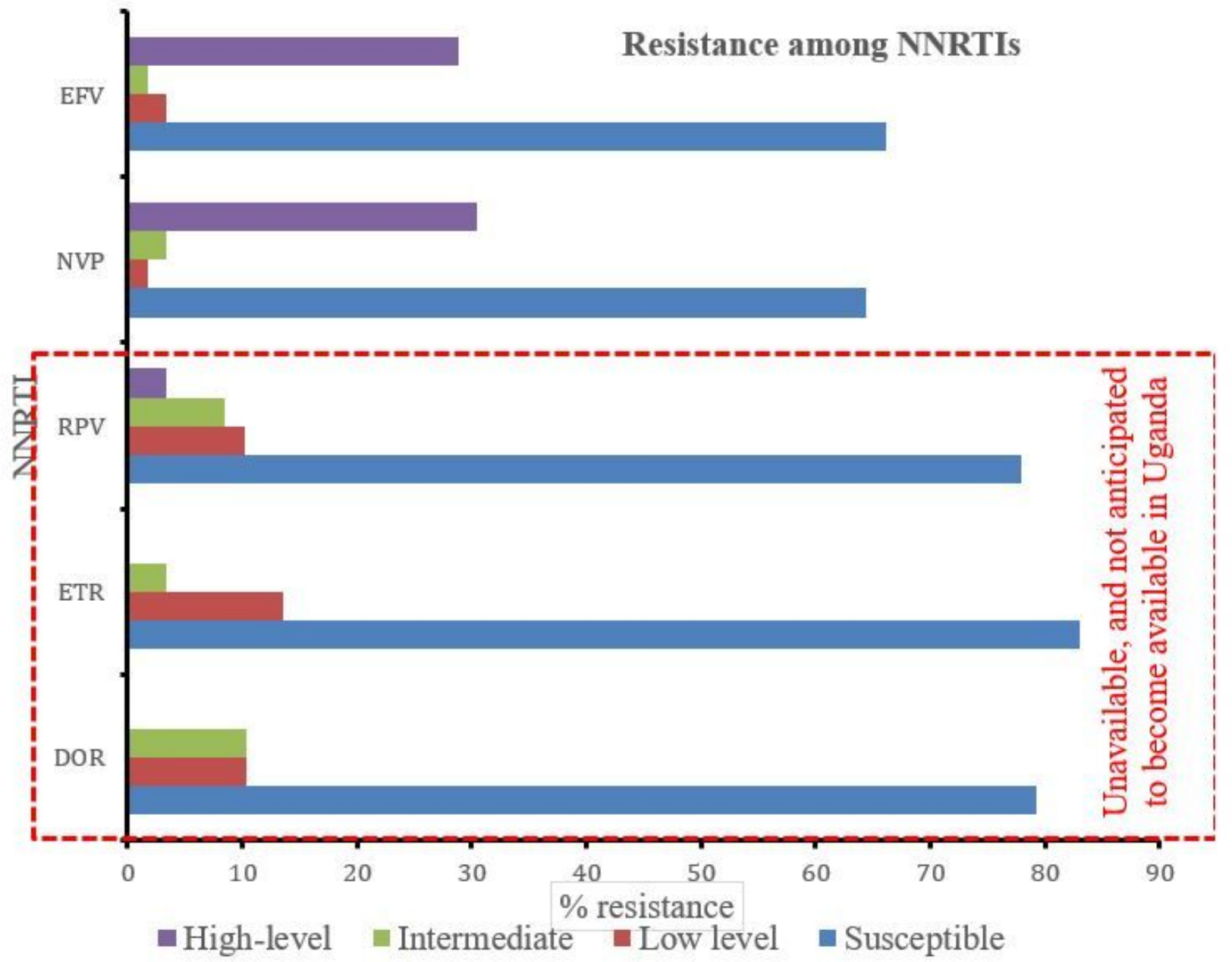

Figure 2

NNRTI resistance among pregnant women living with HIV with high viremia. ETR: etravirine; EFV: EFV; NVP: nevirapine; DOR: doravirine; RPV: rilpivirine. Viral resistance was highest with EFV, nevirapine, and least with etravirine and doravirine. Rilpivirine, dorunavir and etravirine are not options in our population since they are not available. 